\title{
Synergistic effect of radiotherapy and dendritic-cell-based immunotherapy via miR-15b-5p regulation in a colon carcinoma mouse model
}

\author{
Xiaolian Zhang ${ }^{1 \star}$, Wei Wei ${ }^{1}$, LiHong Xia ${ }^{1}$, Ruzhen Zheng ${ }^{2}$ \\ ${ }^{1}$ Department of Radiation Oncology, the First Affiliated Hospital, College of Medicine, Zhejiang University, Hangzhou 310003, \\ ${ }^{2}$ Department of Radiation Oncology, Hangzhou Cancer Hospital, Hangzho, 310002, China
}

*For correspondence: Email: NCarrenosue@yahoo.com; Tel/Fax: 0086-0571-87236114

\begin{abstract}
Purpose: To determine the combined effect of radiotherapy (RT) and dendritic cell (DC)-based immunotherapy on tumor regression, and the influence of miR-15b-5p on the sensitivity of the tumor to RT/DC.

Methods: Colon carcinoma cell line (T-26) was injected subcutaneously into BALB/c mice to generate xenograft tumors. The animals were exposed to radiation therapy (RT) only or DC only, or RT plus DC $(R T / D C)$. Tumor progression was measured and analyzed followed by quantification of miR-15b-5p using qPCR in each group 21 days after tumor inoculation. Overexpressed xenograft tumors were generated by transfecting MiR-15b-5p mimic to CT-26 cell lines and subcutaneously administered to mice. The mice were then subjected to RT/DC treatment and assessed for tumor growth, TUNEL staining, as well as mRNA and protein expressions of B-cell lymphoma-2 (BCL-2), caspase-3 (casp-3), and $X$-linked inhibitor of apoptosis protein (XIAP).

Results: RT/DC combination treatment resulted in tumor regression and increased miR-15b-5p expression, when compared with other groups treated with $R T$ and $D C$ alone $(p<0.001)$. There was a marked reduction in tumor growth following $R T / D C$ combination therapy in miR-15b-5p overexpressed xenograft tumor $(p<0.001)$. Moreover, miR-15b-5p/(RT/DC) treatment significantly increased cancer cell apoptosis. These results were substantiated by increased mRNA and protein expressions of casp-3, reduced $B C L-2$ and XIAP $(p<0.001)$.

Conclusion: These findings demonstrate that RT/DC combination therapy increases miR-15b-5p levels in mice, and that miR-15b-5p upregulation enhances the sensitivity of tumors to RT/DC combination therapy. Therefore, the combination of RT and dendritic cell-based immunotherapy offers significant therapeutic advantage in controlling cancers, including metastatic tumors.
\end{abstract}

Keywords: Radiotherapy, Dendritic cells, Colon carcinoma, Apoptosis, MiR-15b-5p

This is an Open Access article that uses a fund-ing model which does not charge readers or their institutions for access and distributed under the terms of the Creative Commons Attribution License (http://creativecommons.org/licenses/by/4.0) and the Budapest Open Access Initiative (http://www.budapestopenaccessinitiative.org/read), which permit unrestricted use, distribution, and reproduction in any medium, provided the original work is properly credited.

Tropical Journal of Pharmaceutical Research is indexed by Science Citation Index (SciSearch), Scopus, International Pharmaceutical Abstract, Chemical Abstracts, Embase, Index Copernicus, EBSCO, African Index Medicus, JournalSeek, Journal Citation Reports/Science Edition, Directory of Open Access Journals (DOAJ), African Journal Online, Bioline International, Open-J-Gate and Pharmacy Abstracts

\section{INTRODUCTION}

Radiotherapy (RT) is one of the mainstream therapeutic options for cancer. Tumor regression by RT is most often associated with disruption of mitotic processes in cancer cells, thereby causing DNA damage [1-3]. Treatment with RT is not directly linked with early cell death/apoptosis, and it has evolved into a promising immunological adjuvant [4-6]. On the other hand, 
dendritic cells (DCs) are potent immune system regulators. Indeed, dendritic cell-based vaccines have strong implications for the treatment of cancer $[7,8]$. Therefore, the combination of RT and dendritic cell-based immunotherapy may offer great advantage in controlling cancers, including metastatic tumors [9-11]. The molecular mechanism of this combination therapy on tumor regression, and strategies to enhance the immunogenicity of DCs still need extensive investigation.

MicroRNAs (miRNAs) are critical regulators of gene expression which influence cellular growth and death $[12,13]$. It has been postulated that miR-15b-5p acts as a tumor suppressor that negatively regulates post-transcriptional expression of multiple oncogenes [14]. In addition, miR-15b has been studied for its effect on promoting cancer cell apoptosis and inhibiting cellular proliferation, angiogenesis, and metastasis of tumors [14]. Studies have shown that overexpression of miR-15b decreased BCL2 expression and enhanced the activities of the chief apoptosis mediators, caspase-3 and caspase-7 [15]. Moreover, it has been reported that miR-15b upregulation arrested cell cycle at G0/G1 phase, whereas its downregulation promoted S-phase cell proliferation [16]. A study has shown that irradiation-based DNA damage upregulated miR-15b, thereby modulating cellular stress [17]. Moreover, miRNAs are effective tools for characterizing DC maturation and differentiation, and they are beneficial in the assessing the quality of DCs before their clinical use [18]. The present study was carried out to determine the combined effect of radiotherapy (RT) and dendritic cell (DC)-based immunotherapy on tumor regression, and the influence of miR-15b-5p on the sensitivity of the tumor to RT/DC.

\section{EXPERIMENTAL}

\section{Animals}

Ten C57BL/6 mice for bone marrow-derived DC culture, and 35 nude/SCID BALB/c mice for development of colon cancer xenograft model were obtained from SLAC Company, Shanghai, China. The experiments on animals were performed in accordance with the International guidelines for study of laboratory animals [19], and were permitted by the local ethical committee (approval no. SCE20160421).

\section{Bone marrow-derived DC culture}

Bone-marrow cells were acquired from C57BL/6 mice $(n=10)$ as previously described [20]. The
$\mathrm{BM}$ cells were suspended in Tris- $\mathrm{NH}_{4} \mathrm{Cl}$ potassium lysis buffer for $2 \mathrm{~min}$ at room temperature to lyse the red blood cells, followed by centrifugation. The cells were washed in PBS and cultured in RPMI-1640 medium with recombinant mouse interleukin (IL)-4 (10 ng/mL, Thermo Fisher) and $20 \mathrm{ng} / \mathrm{mL}$ recombinant mouse granulocyte-macrophage-colonystimulating factor (Thermo Fisher) at a density of $2 \times 10^{6}$ cells $/ \mathrm{mL}$. After 6 days of culture, nonadherent cells were harvested and observed using FC500.

\section{Colon carcinoma model and treatment}

A colon carcinoma metastatic model was generated using BALB/c mice $(n=35)$. Xenograft tumors were produced by injecting $2 \times 10^{6}$ CT-26 colon carcinoma cells subcutaneously into mice. After seven days, tumor growth, measured as tumour volume $(\mathrm{V})$, was calculated as shown in Eq 1.

$\mathrm{V}=\mathrm{LS}^{2} / 2$

where $L(\mathrm{~mm})$ and $S\left(\mathrm{~mm}^{2}\right)$ are the long and short axes, respectively.

When the tumor volume reached $50-100 \mathrm{~mm}^{3}$ after seven days of inoculation, the mice were divided into four groups: vehicle, radiation alone $(\mathrm{RT})$, intratumoral injection of DCs only (DC), and radiation with $\mathrm{DCs}(\mathrm{RT} / \mathrm{DC})$. Each group was given treatment three times at an interval of 7 days. The optimized dose of radiation i.e. $15 \mathrm{~Gy}$, and $48 \mathrm{~h}$ between radiation and $\mathrm{DC}$ administration were followed. After 21 days, the mice were sacrificed, and the tumor tissues were excised and processed for histological examination.

\section{MiRNA RT-qPCR}

Isolation of total RNA was done using miRVana miRNA isolation kit (Thermo Fisher) based on manufacturer's guidelines. The RNA was reverse-transcribed to cDNA with $\operatorname{TaqMan}^{\circledR}$ MicroRNA Reverse Transcription Kit, and the cDNA was subjected to qPCR using miRVana quantitative RT-PCR primer set for miR-15b-5p (Thermo Fisher). The qPCR was monitored using $\mathrm{ABI}$ PRISM 7000, with U6 RNA as the endogenous control for normalization of mean $\mathrm{Ct}$ values using the $2^{-\Delta \Delta C t}$ method.

\section{MiR-15b-5p mimic transfection}

The MiR-15b-5p mimics and negative control were transiently transfected into CT-26 cells using Lipofectamine RNAiMax (Thermo Fisher, 
USA.), and were harvested at $48 \mathrm{~h}$ following transfection. The stable transfected cells were identified using puromycin $(0.85 \mu \mathrm{g} / \mathrm{mL})$, which was added to the culture medium two days after transfection. The selected puromycin-resistant colonies were expanded for two weeks. Thereafter, xenograft tumors were produced by subcutaneous administration of $2 \times 10^{6} \mathrm{CT}$ $26 /$ vector or CT-26/15b mimic cells. All groups then received $\mathrm{RT} / \mathrm{DC}$ treatment as described previously.

\section{Tumor growth measurement}

Tumor volume was assessed following tumor cell injection every 3 days. Each treatment was started when the measured tumor volumes were $50-100 \mathrm{~mm}^{3}$. Following the first treatment, tumor volumes were continuously measured, and analysed to determine differences between treatment groups. Following 21 days of treatment, the mice were sacrificed, and the tumors were excised and subjected to histological examinations.

\section{TUNEL staining}

The TUNEL staining was executed using ApopTag plus peroxidase in situ apoptosis detection kit (Sigma Aldrich) in line with the protocol described in the kit manual. The tumor samples were fixed in $4 \%$ paraformaldehyde overnight and paraffin-embedded before TUNEL staining.

\section{Determination of mRNA expressions}

Homogenised samples were used to isolate RNA using PureLink RNA mini kit as described in the manual (Thermo Fisher). The concentration of RNA extract was measured using NanoDrop 1000 system (Thermo Fisher). The RNA was converted to cDNA using SuperScript ${ }^{\mathrm{TM}}$ IV VILOTM Master Mix (Thermo Fisher). Then, mRNA expressions of B-cell lymphoma-2 (BCL2), caspase-3 (casp-3), and X-linked inhibitor of apoptosis protein (XIAP) genes were determined with SYBRTM Green PCR Master Mix (Applied Biosystems). Primers were acquired commercially (Thermo Fisher) and GAPDH served as the endogenous control for normalizing the mean Ct values using the $2^{-\Delta \Delta C t}$ method.

\section{Western blot analysis}

Protein was isolated from homogenized tumor samples, and the extracted protein concentration was assayed using BCA kit (Sigma-Aldrich). The protein was subjected to SDS PAGE (10\%), and the separated proteins were transferred to a nitrocellulose membrane using the electroblotting technique. After electro-blotting, the membrane was blocked with $5 \%$ non-fat milk, and incubated overnight at $4{ }^{\circ} \mathrm{C}$ with primary antibodies i.e. rabbit anti-BCL-2 (diluted 1:1000), anti-casp-3 (diluted 1:1000), and anti-XIAP (diluted 1:1000) (Santa Cruz Biotechnology). The membrane was then incubated with goat secondary antibody labeled with horseradish peroxidase (diluted $1: 1000)$ in blocking buffer. The proteins were detected using Chemiluminescence kit (Thermo Fisher Scientific).

\section{Statistical analysis}

All numerical data are represented as the mean \pm standard error of mean (SEM). One-way analysis of variance (ANOVA) was employed to compare the variables followed by Bonferroni post-test and Student's t-test to analyse two independent groups using GraphPad Prism 5.0 for Windows software. The differences were considered statistically significant at $p<0.05$.

\section{RESULTS}

\section{RT/DC combination therapy reduced tumor volume}

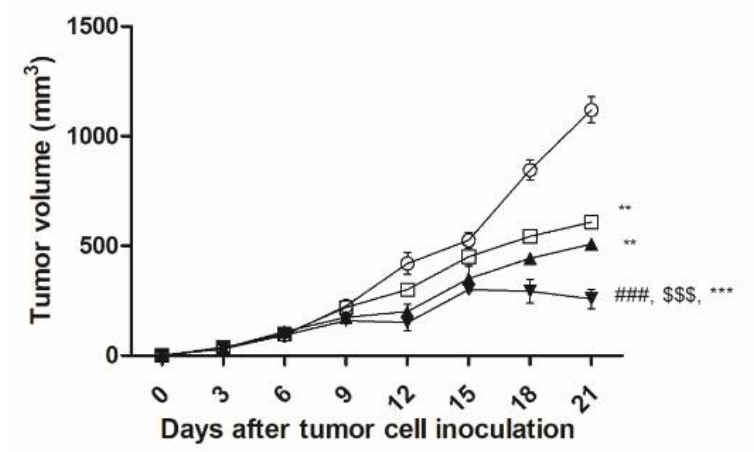

Figure 1: Effect of RT/DC on tumor volume 0 - 21 days after tumor cell inoculation. RT/DC treated tumors showed a remarkably significant tumor regression, when compared to other groups. Data are represented as mean \pm SEM; ${ }^{* *} p<0.01$, compared with vehicle; ${ }^{* \star} p<0.001$, compared to vehicle; ${ }^{\# \# \#} p<$ 0.001 , compared with RT; ${ }^{\$ \$ \$} p<0.001$, compared with DC. The groups are represented by symbols: $0=$ vehicle, $\square=\mathrm{RT}, \boldsymbol{\Delta}=\mathrm{DC}$, and $\boldsymbol{\nabla}=\mathrm{RT} / \mathrm{DC}$

Tumor volume was recorded every 3 days from the day of tumor cell inoculation. Compared to the vehicle-treated group, the other groups showed statistically significant changes in tumor regression over the course of 21 days (Figure 1). The groups subjected to RT or DC treatment only exhibited slight tumor regression, when compared to the vehicle-treated group $(p<0.01)$. 
However, the RT/DC group which received the radiation and intratumoral DC combination therapy showed remarkable decreases in tumor volume, when compared with vehicle group $(p<$ $0.001)$, RT-only group $(p<0.001)$ and DC group $(p<0.001)$.

\section{RT/DC therapy increased miR-15b-5p levels}

To assess the potential function of miR-15b-5p in the RT/DC combination therapy, the miR-15b-5p level was quantified in samples from the three groups: vehicle, RT, DC and RT/DC. The group subjected to RT showed 0.912-fold increase, while the DC group showed 0.81 -fold increase in miR-15b-5p expression, when compared to the vehicle-treated group $(p<0.01)$. The RT/DC group presented a remarkable 2.445-fold increase in miR-15b-5p expression, relative to the vehicle-treated group $((p<0.001$; Figure 2$)$. The change in miR-15b-5p level in the RT/DC group was also significant relative to the RT-only and DC-only groups $(p<0.001)$.

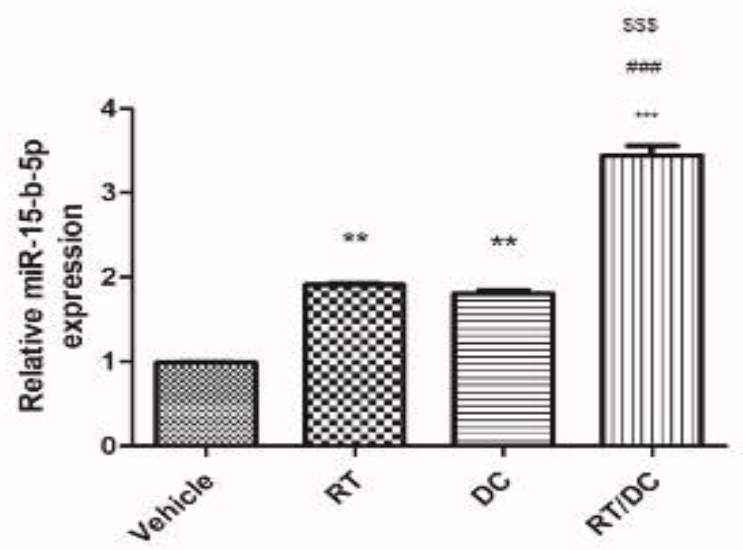

Figure 2: Effect of RT/DC combination on miR-15b-5p expression. Values are presented as mean \pm SEM of fold-change; $p<0.01$, relative to vehicle; $p<0.001$, relative to vehicle; ${ }^{\# \#} p<0.001$, relative to $\mathrm{RT} ;{ }^{\$ \$ \$} p<$ 0.001 , compared to DC

\section{MiR-15b-5p promoted the sensitivity of the tumor to RT/DC treatment}

The upregulation of miR-15b-5p enhanced the sensitivity of the tumor to RT/DC treatment, resulting in a remarkable decrease in tumor volume from day 9 of tumor cell inoculation, up to the $21^{\text {st }}$ day, relative the vector/untreated group $(p<0.001)$. The vector/(RT/DC) group and miR$15 \mathrm{~b}-5 \mathrm{p} /$ untreated group showed decreases in tumor volume, when compared with the vector/untreated group. Thus, miR-15b-5p upregulation directly enhanced the sensitivity of tumors to RT/DC.

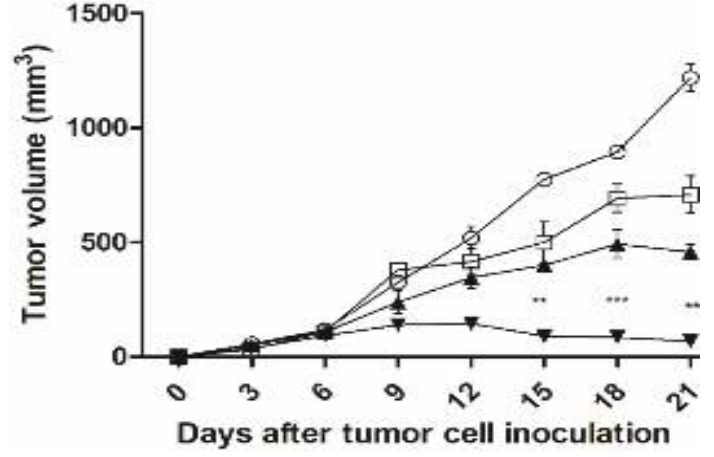

Figure 3: Effect of RT/DC on tumors with miR-15b-5p overexpression. Treatment with miR-15b-5p/(RT/DC) resulted in a significant tumor regression starting from day 9 , indicating the relevance of miR-15b-5p upregulation in enhancing the sensitivity of tumors to $\mathrm{RT} / \mathrm{DC}$ treatment. Values are presented as mean \pm SEM; ${ }^{* *} p<0.001,{ }^{* *} p<0.01$, compared with vector/untreated. The groups are represented by symbols: $\circ=$ vector/untreated, $\square=$ miR-15b$5 \mathrm{p} /$ untreated, $\boldsymbol{\Delta}=$ vector/ $(\mathrm{RT} / \mathrm{DC})$, and $\boldsymbol{\nabla}=\mathrm{miR}-15 \mathrm{~b}-$ $5 \mathrm{p} /(\mathrm{RT} / \mathrm{DC})$

\section{MiR-15b-5p upregulation promoted cell death in RT/DC-treated tumor}

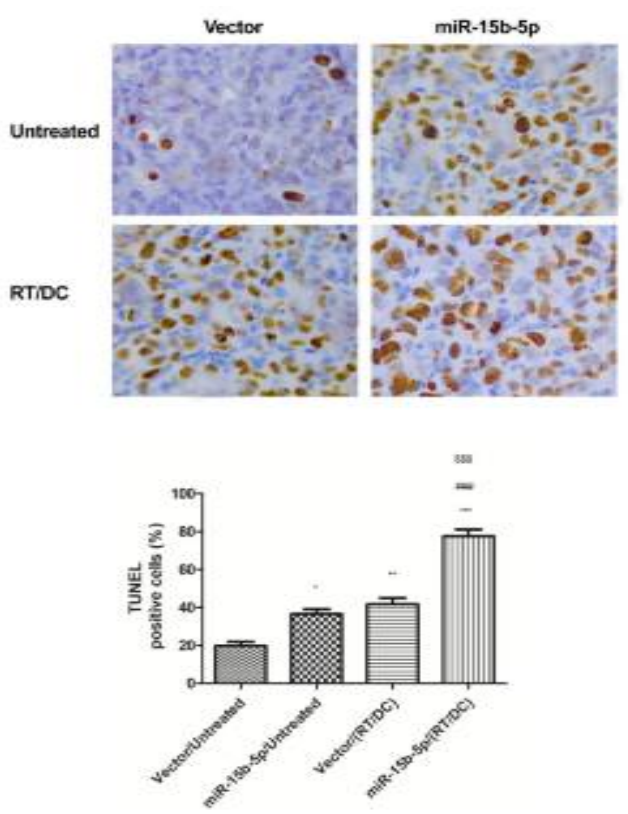

Figure 4: Effects of RT/DC and MiR-15b-5p overexpression on apoptosis. (a) Representative images of TUNEL-stained cells in vector/untreated, vector/(RT/DC), miR-15b-5p/untreated, and miR-15b$5 \mathrm{p} /(\mathrm{RT} / \mathrm{DC})$ cells $(\times 40)$. (b) Percentage of TUNELpositive cells (\%). " $p<0.05,{ }^{* *} p<0.01,{ }^{* \star *} \quad p<0.001$, compared with vehicle/untreated; \#\# $p<0.001$ compared to vector/(RT/DC), and $\$ \$ p<0.001$, compared to miR-15b- $5 p /$ untreated

TUNEL staining demonstrated that miR-15b-5p upregulation significantly increased cell death in tumor samples treated with RT/DC (Figure 3a, \& 
$3 b)$. The average cell death increased by $57.8 \%$ in $\mathrm{miR}-15 \mathrm{~b}-5 \mathrm{p} /(\mathrm{RT} / \mathrm{DC})$ group, when compared to other groups $(p<0.001)$. Vector/(RT/DC) and miR-15b-5p/untreated groups also exhibited significant increases in cell death $(20.9 \%, p<$ 0.01 vs vector/untreated; and $16.76 \%, p<0.05$ vs vector/untreated, respectively). These results imply that RT/DC and miR-15b-5p also had individual effects on cell death.

\section{MiR-15b-5p promoted the expression of cell death-related genes in RT/DC-treated tumor sample}

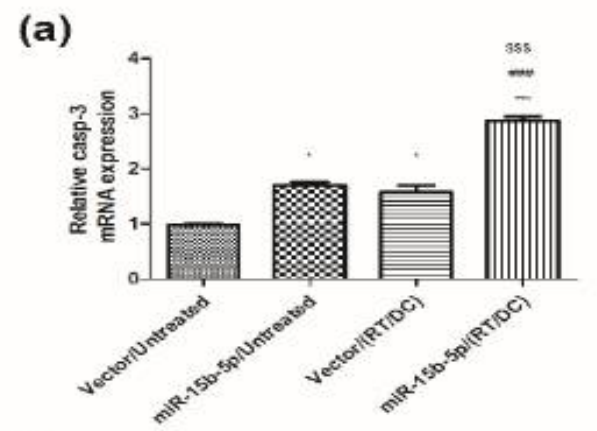

(b)

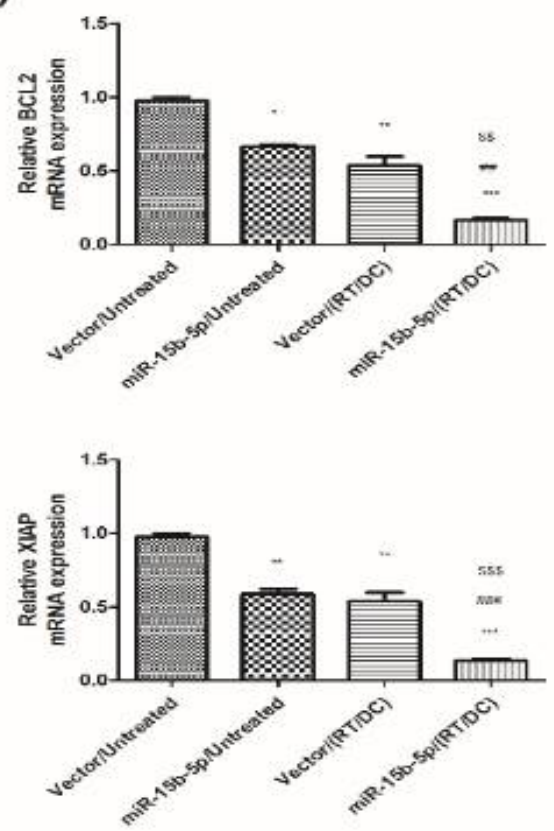

Figure 5: Effect of RT/DC on relative mRNA expression of apoptosis-linked genes. (a) Casp-3, (b) $\mathrm{BCL}-2$, and (c) XIAP. Values are presented as mean fold-change $\pm \mathrm{SEM}_{2}$ relative to endogenous control GAPDH. $p<0.05, \quad p<0.01, \quad p<0.001$, compared to vehicle/untreated; $p<0.001$, compared to vector/(RT/DC), and $\$ \$ p<0.001, \$ p<0.01$, compared to miR-15b-5p/untreated

To further investigate the mechanism involved in the enhancement of the sensitivity of tumors to RT/DC combination treatment by miR-15b-5p upregulation, mRNA expression of the apoptosis- associated genes i.e. casp-3, BCL-2 and XIAP were determined. Treatment with miR-15b$5 p /(R T / D C)$ resulted in a significant 1.86 -fold rise in casp-3, 0.834-fold decrease in BCL-2, and 0.864 -fold decrease in XIAP mRNA expressions, when compared to vector/untreated group ( $p<$ 0.001; Figures 5a, $5 \mathrm{~b}$ and 5c). However, significant fold-changes were seen in casp-3, BCL-2 and XIAP expressions, relative to the miR-15b-5p/untreated and vector/(RT/DC) groups ( $p<0.001$, compared to vector/(RT/DC); $p<0.001$, compared to miR-15b-5p/untreated) (Figures 5a, 5b and 5c). The corresponding changes in miR-15b-5p/untreated and vector/(RT/DC) groups were marginal, when compared to miR-15b-5p/(RT/DC), indicating the potential apoptosis-modulating effect of miR-15b$5 p /(R T / D C)$.

\section{Verified levels of apoptosis-linked proteins casp-3, BCL-2, and XIAP}

Western blot confirmed the GPCR mRNA expressions of casp-3, BCL-2 and XIAP in miR15b-5p-upregulated, RT/DC-treated tumors. There were decreases in BCL-2 and XIAP mRNA, whereas casp-3 increased in miR-15b-5/(RT/DC) group. Altered expressions of these proteins in miR-15b-5p/untreated and vehicle/(RT/DC) groups were observed. However, the effect was maximum in the miR$15 b-5 p /(R T / D C)$ group. These results indicate that miR-15b-5p upregulation enhanced the sensitivity of the tumor to RT/DC treatment.

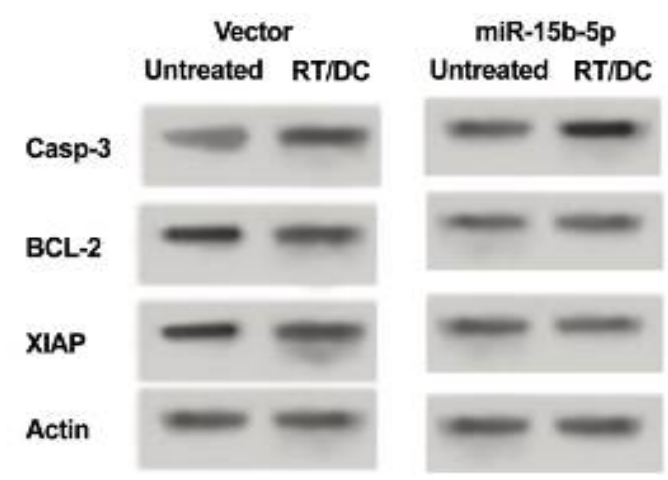

Figure 6: Western blot results for apoptosis-linked proteins casp-3, BCL-2, and XIAP. The protein expression analysis for vector/untreated, vector/(RT/DC), miR-15b-5p/untreated and miR-15b$5 \mathrm{p} /(\mathrm{RT} / \mathrm{DC})$ is shown. Casp-3 expression increased, whereas BCL-2 and XIAP protein expressions decreased markedly in miR-15b-5p/(RT/DC) group, relative to the other groups

\section{DISCUSSION}

The present study demonstrates the synergistic effect of RT and DC-based immunotherapy on 
tumor regression with mechanistic insight on the function of miR-15b-5p in enhancing the sensitivity of tumor to RT/DC combination treatment. The understanding of immune mechanisms and the implications of RT on tumor microenvironment has resulted in the development of innovative treatment approaches for cancer. Many reports support the use of combination of RT and immunotherapy to achieve a better local/systemic control of tumors [9-11]. The irradiation of tumor predominantly leads to DNA damage, resulting mitotic catastrophe which is not directly linked to premitotic cell death or apoptosis [4,5]. The RTinduced DNA damage activates the immune regulatory mechanism. Accumulating evidence show that the immune system of the host generates cytotoxic adaptive response which enables immunogenic cell death and tumor regression [21]. The efficiency of immunogenic cell death also depends on tumor type and immunogenic capacity, while RT enhances this fine-tuned process via the activation of critical inflammatory processes [21].

The preparation of DCs with tumor-associated antigens (TAAs) is crucial for generating DC vaccines for the induction of immunotherapy in tumors, and it can be attained by culturing DCs with irradiated cancer cells ex vivo or in vivo [22]. The irradiation of cancer cells promotes the generation of TAAs and increases the stimulation of DCs. A study has reported thaty-irradiation of DCs resulted in enhanced anti-cancer activity by increasing the cytotoxicity and activation of $T$ cells [23]. In view of extant studies on the role of DCs against cancer and the influence of RT as an immunological adjuvant, the effect of RT/DC combination therapy compared with sole treatment with RT or DC, was investigated in the present study in a colon carcinoma metastatic mouse model. The findings showed that RT/DC combination treatment resulted in remarkably significant tumor regression, when compared with other groups. However, the application of RT/DC therapy in cancer is still in its infancy. Extensive investigations are required on ways to enhance the immunogenicity of DCs and sensitization of tumor to the treatment.

Furthermore, the regulatory function of miR-15b$5 p$ following RT/DC combination therapy was assessed. It is known that miR-15b which is aberrantly expressed in malignant tumors, is involved in cancer progression [14]. The upregulation of miR-15b induces cell death, reduces cancer cell proliferation, and decreases angiogenesis and metastasis of tumors [14]. In the present study, miR-15b-5p was significantly downregulated in the untreated group, while the
RT/DC treatment significantly increased miR$15 b-5 p$ level, relative to the other groups. This observation led to investigations on the upregulation of miR-15b-5p in colon carcinoma model in-vivo, and its effect on the sensitivity of tumor to RT/DC treatment.

Another objective of this study was to investigate the impact of miR-15b-5p upregulation on tumor regression following $\mathrm{RT} / \mathrm{DC}$ treatment. The results showed that miR-15b-5p mimic and RT/DC treatment had individual influences on tumor regression and cellular apoptosis. However, the effect was remarkably enhanced in the group treated with both miR-15b-5p mimic and $\mathrm{RT} / \mathrm{DC}$ combination. A recent study reported that miR-15b-5p upregulation enhanced 5fluorouracil-induced cell death in a colorectal cancer model. MiR-15b-5p mimic transfection also reversed the chemo-resistance of cancer cells to 5-fluorouracil via NFkB1 signaling pathway [24].

Therefore, in this study, the marked reduction in tumor growth and enhanced apoptosis of cancer cells imply that miR-15b-5p upregulation indeed promotes the sensitivity of tumors to RT/DC treatment. It has been reported that miR-15b-5p directly targeted $\mathrm{BCL}-2$, and that increases in miR-15b-5p reduced $B C L-2$ while increasing casp-3 and casp-7 protein levels [15]. A previous study showed that inhibition of miR-15b attenuated TRAIL-mediated apoptosis, whereas miR-15b precursor increased cell death [25]. Another study demonstrated that administration of dihydroartemisinin for the treatment of gastric cancer resulted in an increased miR-15b expression concomitant with reduced expression of BCL-2 [26]. Moreover, increased XIAP level has been associated with progression of cancer, and miR-15b-5p mimic was reduced with XIAP expression, thereby promoting apoptosis of cancer cells [24]. The results obtained in the present study, wherein miR-15b-5p upregulation resulted in a significant increase in casp-3 and marked reductions in BCL-2 and XIAP levels in $\mathrm{RT} / \mathrm{DC}$ treated group, are consistent with these findings.

\section{CONCLUSION}

The findings of this study show, for the first time, that RT/DC combination therapy increases miR$15 b-5 p$ expression. Furthermore, miR-15b-5p upregulation enhances the sensitivity of tumors to RT/DC combination therapy via the regulation of genes and proteins associated with cancer cell apoptosis. Thus, this study opens a new avenue for optimization of DC preparation and RT, and enhancing the immunogenicity of the DCs. 


\section{DECLARATIONS}

\section{Conflict of interest}

No conflict of interest is associated with this work.

\section{Contribution of authors}

We declare that this work was done by the authors named in this article and all liabilities pertaining to claims relating to the content of this article will be borne by the authors.

\section{Open Access}

This is an Open Access article that uses a funding model which does not charge readers or their institutions for access and distributed under the terms of the Creative Commons Attribution License (http://creativecommons.org/licenses/by/ 4.0) and the Budapest Open Access Initiative (http://www.budapestopenaccessinitiative.org/rea d), which permit unrestricted use, distribution, and reproduction in any medium, provided the original work is properly credited.

\section{REFERENCES}

1. Delaney G, Jacob S, Featherstone C, Barton M. The role of radiotherapy in cancer treatment: estimating optimal utilization from a review of evidence-based clinical guidelines. Cancer 2005; 104: 1129-1137.

2. Eriksson $D$, Stigbrand T. Radiation-induced cell death mechanisms. Tumour Biol 2010; 31: 363-372.

3. lanzini $F$, Bertoldo A, Kosmacek EA, Phillips SL, Mackey MA. Lack ofp53 function promotes radiation-induced mitotic catastrophe in mouse embryonic fibroblast cells. Cancer Cell Int 2006; 6: 11.

4. Dewey WC, Ling CC, Meyn RE. Radiation-induced apoptosis: relevance to radiotherapy. Int $J$ Radiat Oncol Biol Phys 1995; 33: 781-796.

5. Roos WP, Thomas AD, Kaina B. DNA damage and the balance between survival and death in cancer biology. Nat Rev Cancer 2016; 16: 20-33.

6. Demaria S, Formenti SC. Radiation as an immunological adjuvant: current evidence on dose and fractionation. Front Oncol 2012; 2: 153.

7. Kalinski P, Urban J, Narang R, Berk E, Wieckowski E, Muthuswamy R. Dendritic cell-based therapeutic cancer vaccines: what we have and what we need. Future Oncol 2009; 5: 379-390.

8. Santos PM, Butterfield LH. Dendritic Cell-Based Cancer Vaccines. J Immunol 2018; 200: 443-449.

9. Tang $C$, Wang $X$, Soh $H$, Seyedin $S$, Cortez $M A$, Krishnan S, Massarelli E, Hong D, Naing A, Diab A, et al. Combining radiation and immunotherapy: a new systemic therapy for solid tumors? Cancer Immunol Res 2014; 2: 831-838.

10. Bockel S, Durand B, Deutsch E. Combining radiation therapy and cancer immune therapies: From preclinical findings to clinical applications. Cancer Radiother 2018; pii: S1278-3218(18)30300-7.

11. Wang $Y$, Deng W, Li N, Neri S, Sharma A, Jiang W, Lin $\mathrm{SH}$. Combining Immunotherapy and Radiotherapy for Cancer Treatment: Current Challenges and Future Directions. Front Pharmacol 2018; 9: 185.

12. Lee YS, Dutta A. MicroRNAs in cancer. Annu Rev Pathol 2009; 4: 199-227

13. Adams BD, Kasinski AL, Slack FJ. Aberrant regulation and function of microRNAs in cancer. Curr Biol 2014; 24: $R 762-776$.

14. Zhao $C$, Wang G, Zhu Y, Li X, Yan F, Zhang $C$, Huang $X$, Zhang $Y$. Aberrant regulation of miR-15b in human malignant tumors and its effects on the hallmarks of cancer. Tumour Biol 2016; 37: 177-183.

15. Xia L, Zhang D, Du R, Pan Y, Zhao L, Sun S, Hong L, Liu $J$, Fan $D$. Mir-15b and mir-16 modulate multidrug resistance by targeting bcl2 in human gastric cancer cells. Int J Cancer 2008; 123: 372-379.

16. Xia H, Qi Y, Ng SS, Chen X, Chen S, Fang M, Li D, Zhao $Y, G e R, L i G$, et al. MicroRNA-15b regulates cell cycle progression by targeting cyclins in glioma cells. Biochem Biophys Res Commun 2009; 380: 205-210.

17. Rahman M, Lovat F, Romano G, Calore F, Acunzo M, Bell EH, Nana-Sinkam P. miR-15b/16-2 regulates factors that promote $p 53$ phosphorylation and augments the DNA damage response following radiation in the lung. J Biol Chem 2014; 289: 26406-26416.

18. Holmstrøm K, Pedersen AW, Claesson MH, Zocca MB, Jensen SS. Identification of a microRNA signature in dendritic cell vaccines for cancer immunotherapy. Hum Immunol 2010; 71: 67-73.

19. Garber JC (Chair). Committee for the Update of the Guide for the Care and Use of Laboratory Animals. Guide for the Care and Use of Laboratory Animals, eighth ed. Washington DC, USA: National Academy of Sciences; 2011.

20. Wang W, Li J, Wu K, Azhati B, Rexiati M. Culture and Identification of Mouse Bone Marrow-Derived Dendritic Cells and Their Capability to Induce $T$ Lymphocyte Proliferation. Medical Science Monitor: Int Med J Exper Clin Res 2016; 22: 244-250.

21. Schaue $D, M c B r i d e ~ W H$. Links between innate immunity and normal tissue radiobiology. Radiat Res 2010; 173: 406-417.

22. Chi KH, Liu SJ, Li CP, Kuo HP, Wang YS, Chao Y, Hsieh $S L$. Combination of conformal radiotherapy and intratumoral injection of adoptive dendritic cell immunotherapy in refractory hepatoma. $J$ Immunother 2005; 28: 129-135.

23. Kim SK, Yun $\mathrm{CH}$, Han SH. Enhanced anti-cancer activity of human dendritic cells sensitized with gammairradiation-induced apoptotic colon cancer cells. Cancer Lett 2013; 335: 278-288.

Trop J Pharm Res, May 2019; 18(5): 947 
24. Zhao $C$, Zhao $Q$, Zhang $C$, Wang $G$, Yao $Y$, Huang $X$, Zhan F, Zhu $Y$, Shi J, Chen J, et al. miR-15b-5p resensitizes colon cancer cells to 5-fluorouracil by promoting apoptosis via the NF-KB/XIAP axis. Sci Rep 2017; 7: 4194.

25. Chung GE, Yoon JH, Myung SJ, Lee JH, Lee SH, Lee SM, Kim SJ, Hwang SY, Lee HS, Kim CY. High expression of microrna-15b predicts a low risk of tumor recurrence following curative resection of hepatocellular carcinoma. Oncol Rep 2010; 23: 113-119.

26. Sun H, Meng X, Han J, Zhang Z, Wang B, Bai X, Zhang $X$. Anti-cancer activity of dha on gastric cancer-an in vitro and in vivo study. Tumour Biol 2013; 34: 37913800 . 